\title{
Cardiovascular effects of epidural morphine or ropivacaine in isoflurane-anaesthetised pigs during surgical devascularisation of the liver
}

\author{
G F Stegmann
}

\begin{abstract}
The cardiovascular effects of non-abdominal and abdominal surgery during isoflurane anaesthesia (A-group) or isoflurane anaesthesia supplemented with either epidural ropivacaine (AR-group; $0.75 \%$ solution, $0.2 \mathrm{~m} / \mathrm{kg}$ ) or morphine (AM-group; $0.1 \mathrm{mg} / \mathrm{kg}$ diluted in saline to $0.2 \mathrm{~m} / \mathrm{kg}$ ) were evaluated in 28 healthy pigs with a mean body weight of $30.3 \mathrm{~kg} \mathrm{SD} \pm 4.1$ during surgical devascularisation of the liver. Anaesthesia was induced with the intramuscular injection of midazolam $(0.3 \mathrm{mg} / \mathrm{kg})$ and ketamine $(10 \mathrm{mg} / \mathrm{kg})$. Anaesthesia was deepened with intravenous propofol to enable tracheal intubation and maintained with isoflurane on a circle rebreathing circuit. The vaporiser was set at $2.5 \%$ for the A-group and $1.5 \%$ for the AR- and AM-groups. Differences between treatment groups were not statistically significant $(P>0.05)$ for any of the variables. Differences between AMand AR-groups were marginally significant heart rate $(\mathrm{HR})(P=0.06)$ and mean arterial blood pressure (MAP) $(P=0.08)$. Within treatment groups, differences for the A-group were statistically significant $(P<0.05)$ between non-abdominal and abdominal surgery for $\mathrm{HR}$, systolic blood pressure, diastolic blood pressure (DIA) and MAP. Within the AM-group differences were statistically significant $(P<0.05)$ for DIA and MAP, and within the AR group differences for all variables were not statistically significant $(P>0.05)$. It was concluded that in isoflurane-anaesthetised pigs, the epidural administration of ropivacaine decreased heart rate and improved arterial blood pressure during surgery.
\end{abstract}

Keywords: anaesthesia, cardiovascular, epidural, morphine, pigs, ropivacaine.

Stegmann G F Cardiovascular effects of epidural morphine or ropivacaine in isofluraneanaesthetised pigs during surgical devascularisation of the liver. Journal of the South African Veterinary Association (2010) 81(3): 143-147 (En.). Department of Companion Animal Clinical Studies, Faculty of Veterinary Science, University of Pretoria, Private Bag X04, Onderstepoort, 0110 South Africa.

\section{INTRODUCTION}

In a research project aimed at the development of a bioartificial liver support system (BALSS) using the pig as model, it became essential to ensure optimal perioperative cardiovascular stability to be able to evaluate the BALSS ${ }^{19}$. Surgery may be performed using either regional anaesthetic techniques such as epidural anaesthesia or general anaesthesia ${ }^{7}$. The combined use of regional anaesthesia and general anaesthesia has increased in popularity for procedures involving major intra-abdominal surgery in humans $\mathrm{s}^{2,23}$ and $\operatorname{dogs} \mathrm{s}^{13}$. Important perioperative advantages of the combined administration are a reduction in the minimum alveolar concentration of inhalation anaesthetics $^{2,17}$, postoperative analgesia ${ }^{13}$ and improved morbidity/mortality rates ${ }^{21}$. The perioperative use of epidural anaes-

${ }^{a}$ Department of Companion Animal Clinical Studies, Faculty of Veterinary Science, University of Pretoria Private Bag X04, Onderstepoort, 0110 South Africa

E-mail: frik.stegmann@ up.ac.za

Received: May 2009. Accepted: May 2010. thesia on its own or in combination with general anaesthesia remain a controversial issue as adverse effects such as bradycardia and hypotension have been reported in humans ${ }^{1,18}$ and animals ${ }^{26}$ despite potential long-term advantages such as improved postoperative recovery $^{18,21}$. Adverse effects associated with surgery with particular reference to the stress response may be reduced by the use of either spinal/epidural anaesthesia ${ }^{8,9,28}$ or the local infiltration of lidocaine and bupivacaine before laparotomy ${ }^{16}$.

Possible measures to reduce perioperative hypotension were to replace the local anaesthetic with an opioid during epidural anaesthesia and pre-induction fluid administration ${ }^{18}$. Epidural local anaesthetics block nociceptive (sensory), motor and autonomic (sympathetic) pathways whereas opioids such as morphine only affect the nociceptive component of sympathetic activation ${ }^{18}$. Analgesic effects of local anaesthetics may result not only from the spinal root blockade of sensory nerves but also from systemic effects after epidural absorption ${ }^{8}$.

Potential benefits from the combined use of epidural anaesthesia and general anaesthesia appropriate to the development of a BALSS includes increased cardiovascular stability associated with reduced blood loss, improved vascular graft patency, reduced coronary vasoconstriction and improved intestinal oxygenation $^{8,27}$. In a previous investigation the epidural administration of ropivacaine during surgical removal of the liver in isoflurane-anaesthetised pigs was associated with a decrease in heart rate ${ }^{25}$.

The purpose of this investigation was to evaluate the effects of abdominal surgery on cardiovascular parameters during isoflurane anaesthesia on its own or isoflurane anaesthesia supplemented with either epidural morphine or epidural ropivacaine.

\section{MATERIALS AND METHODS}

Twenty-eight female Landrace pigs with a mean body weight of 30.3 $(\mathrm{SD} \pm 4.1) \mathrm{kg}$ were used in this investigation. The pigs originated from the same breeder and were approximately 3-4 months old. The pigs were part of a study to develop a BALSS, and scheduled for surgical devascularisation of the liver. Each treatment was administered in a block, i.e. 10 pigs for isoflurane anaesthesia only (A-group), 10 pigs for anaesthesia plus ropivacaine epidural (AR-group) and then 8 pigs for anaesthesia plus morphine epidural (AM-group). Two pigs from the latter group were excluded due to failure to catheterise the medial saphenous artery for arterial blood pressure measurement. Surgery of the liver included ligation of the hepatic artery, dissection and isolation of the portal vein and temporary occlusion of the portal vein during surgical anastomosis to the caudal vena cava. Approval for the study was obtained from the Animal Use and Care Committee of the University of Pretoria.

The pigs were deprived of solid food for 24 hours before anaesthesia with water ad libitum. Drinking water was supple- 
mented with dextrose and electrolytes mixed into the water (Rehidrat, Pfizer) during the day (08:00-16:00). At 16:00, $1 \mathrm{~g}$ of a broad-spectrum antibiotic ceftriaxone (Rociject, Sandoz) and $2 \mathrm{mg}$ of a longacting sedative, lorazepam (Ativan, Aspen) was administered intramuscular (IM). Thereafter they were deprived of water as well until induction of anaesthesia the following morning.

The pigs were induced in the pens with the IM injection of midazolam $(0.3 \mathrm{mg} / \mathrm{kg}$, Dormicum, Roche) and ketamine (10 mg/kg, Anaket, Centaur) and moved to theatre. A 20G teflon catheter (Jelco, Johnson \& Johnson) was placed in the ear vein. Anaesthesia was deepened with the intravenous (IV) injection of propofol (Diprivan, Astra Zeneca, $3 \mathrm{mg} / \mathrm{kg}$ ) administered to effect for tracheal intubation with a $7.5 \mathrm{~mm}$ cuffed tracheal tube. Anaesthesia was maintained with an air-oxygen mixture delivered by a precision vaporiser (Isotec 5, Ohmeda). For the A-group, the vaporiser was set at $2.5 \%$ isoflurane and for the AM- and AR-groups the vaporiser was set at $1.5 \%$ isoflurane. A circle rebreathing circuit with carbon dioxide $\left(\mathrm{CO}_{2}\right)$ absorption was used to deliver the inhalation anaesthetic. Fresh gas flow rate was set at $300 \mathrm{~m} / /$ min for oxygen and $600 \mathrm{~m} / / \mathrm{min}$ for air. Minute volume was maintained with positive pressure ventilation (Ohmeda 7000 Ventilator) to maintain end-tidal $\mathrm{CO}_{2}$ partial pressure at $5.3 \mathrm{kPa}$. Core body temperature was maintained with a forced hot air blanket (Bair Hugger 505, Augustine Medical).

The lumber epidural block was administered after tracheal intubation and before surgical preparation. A 20G spinal needle (Vygon) was introduced between lumbar vertebrae 1 and 2 using either a $7.5 \mathrm{mg} / \mathrm{m}$ ropivacaine solution $(0.2 \mathrm{~m} / \mathrm{kg}$, Naropin, Astra) or preservative-free morphine sulphate at $0.1 \mathrm{mg} / \mathrm{kg}$ (Morphine sulphate, Fresenius Kabi) diluted in sterile saline to a volume of $0.2 \mathrm{~m} / \mathrm{kg}$. Placement of the needle tip in the epidural space was detected by the loss of resistance to saline injection. In the A-group, epidural ropivacaine was administered at the completion of surgery for postoperative analgesia (similar to the AR-group). Anaesthesia was maintained postoperatively for $24 \mathrm{~h}$ in all pigs with the IV infusion of fentanyl, pentobarbitone and midazolam $^{19}$.

Cardiovascular volume and blood glucose concentration were maintained with $60 \mathrm{ml}$ of a $50 \%$ dextrose solution added to $1000 \mathrm{~m} \ell$ of a balanced electrolyte solution (Intramed, Ringer Lactate) and administered IV at a rate of $15-30 \mathrm{~m} / \mathrm{kg} / \mathrm{hr}$ for the duration of anaesthesia. During abdominal surgery $20 \mathrm{~m} / \mathrm{kg}$ of a gelatin plasma-expander (Gelofusine, B/Braun) was administered IV.

The medial saphenous artery was percutaneously catheterised using the Seldinger technique with a $20 \mathrm{G}$ polyurethane catheter (Arterial Catheterisation Set, Arrow) for intraoperative arterial blood pressure monitoring. Following epidural administration, the pigs were surgically prepared for abdominal surgery and catheterisation of the internal and external jugular veins, and the carotid artery. Surgery started approximately $30 \mathrm{~min}$ after induction of anaesthesia. For central venous pressure (CVP) measurement a 7 Fr. multiple lumen central venous catheter (Arrow) was introduced in the internal jugular vein after surgical exposure of the jugular groove. Placement of the catheter tip in the right atrium was verified by pressure and waveform changes during catheter introduction.

Cardiopulmonary parameters were monitored with a multi-function patient monitor (TL-101T, Nihon Kohden, Medical Systems). Heart rate (HR), systolic blood pressure (SYS), diastolic blood pressure (DIA), mean arterial blood pressure (MAP) and CVP were monitored. Monitoring of parameters started $5 \mathrm{~min}$ before start of non-abdominal surgery and was recorded at $5 \mathrm{~min}$ intervals. For purposes of this investigation, time points $1-5$ represent the last 5 values during nonabdominal surgery and 6-10 the first 5 values during abdominal surgery (Fig. 1). The value at time point 10 was before temporary occlusion of the portal vein. The $\mathrm{CO}_{2}$ censor for the capnometer was placed between the tracheal tube and the anaesthetic breathing circuit; the electrocardiograpic leads were attached to the limbs; the pulse oximeter probe attached to the tongue; and arterial blood pressure measured from the medial saphenous artery using an electronic pressure transducer (MX 950 Transtar Pressure Transducers, Medex Medical). Anaesthetic depth during surgery was evaluated from the palpebral- and pedal reflex, and the masseter muscle tone. The pedal reflex was evaluated in the

Table 1: Mean \pm SD cardiovascular variables for isoflurane-anaesthetised pigs supplemented with epidural morphine or epidural ropivacaine during surgery.

\begin{tabular}{lccc}
\hline Variable & An & An+M & An+R \\
\hline HR (beats/min) & $90.2 \pm 13.3$ & $99.4 \pm 19.5$ & $79.5 \pm 19.0$ \\
SYS $(\mathrm{kPa})$ & $13.8 \pm 1.8$ & $13.7 \pm 2.6$ & $14.8 \pm 2.3$ \\
DIA $(\mathrm{kPa})$ & $8.7 \pm 1.7$ & $8.7 \pm 1.9$ & $9.8 \pm 1.6$ \\
MAP $(\mathrm{kPa})$ & $10.9 \pm 1.9$ & $10.7 \pm 1.9$ & $12.0 \pm 1.7$ \\
CVP $(\mathrm{kPa})$ & $2.2 \pm 0.3$ & $2.3 \pm 0.4$ & $2.1 \pm 0.5$ \\
\hline
\end{tabular}

$\mathrm{HR}$, heart rate; SYS, systolic pressure; DIA, diastolic pressure; MAP, mean arterial pressure; CVP, central venous pressure; $A n$, anaesthesia; $A n+M$, anaesthesia + epidural morphine; $A n+R b$, anaesthesia + epidural ropivacaine.

front limb by applying pressure to the interdigital skin with tissue forceps for 1 second.

\section{Data analysis}

Data are presented as the mean \pm standard deviation of the mean $( \pm S D)$. Data were tested for normality of distribution (Mauchly's test) and homogeneity of variance (Levene's test). When the variance-covariance matrix of the dependent variables was not circular in form the Greenhouse-Geisser epsilon adjustment for the degrees of freedom was applied. A general linear model for repeated measures was used to analyse the data for differences within treatments groups (non-abdominal and abdominal surgery) and between treatment groups. Where statistically significant differences between treatment groups were observed, Dunnett's T3 post hoc test was used for data that were not normally distributed. Statistical significance was set at $P<0.05$. A statistical software package SPSSVersion 17 (SPSS Inc., Chicago, Illinois) for a personal computer was used to analyse data.

\section{RESULTS}

Data were not normally distributed and the Greenhouse Geiser adjustment for degrees of freedom was used for the average tests of significance. Variances were equal across treatment groups $(P>$ $0.05)$. During surgery the palpebral and pedal reflexes remained absent and the masseter muscles relaxed in all animals. The mean \pm SD cardiovascular values over time (1-10) are graphically presented in Fig. 1a-e. The mean \pm SD values for the treatment groups are reported in Table 1 . The mean $\pm \mathrm{SD}$ values for procedure, i.e. non-abdominal surgery (1-5) and abdominal surgery (6-10), and statistical significance of differences within groups (i.e. between procedures) are reported in Table 2 . The mean \pm SD blood loss during surgery for the A-, AM- and AR-groups were $63 \pm 31 \mathrm{~m} \ell, 25.6 \pm 14.2 \mathrm{~m} \ell$ and $62.2 \pm 45.8 \mathrm{ml}$ respectively. During anaesthesia $1 \ell$ of the balanced electrolyte solution and $500 \mathrm{~m}$ lof the colloid solution was administered to each pig. 
Overall the differences between treatments were not statistically significant $(P=0.11)$. The differences between the individual variables were statistically significant for HR $P=0.017$, DIA $P=0.04$ and MAP $P=0.039$, but not for SYS and $\operatorname{CVP}(P=0.37$ and $P=0.3$ respectively). The post hoc tests indicated no statistically significant difference between treatments for any of the variables: HR, A-AM $P=$ 0.41 ; A-AR $P=0.2$; AM-AR $P=0.06$; SYS, $\mathrm{A}-\mathrm{AM} P=1.0$; A-AR $P=0.48$; AM-AR $P=$ 0.65; DIA, A-AM $P=1.0$; A-AR $P=0.1$; AM-AR $P=0.12$, MAP, A-AM $P=0.99$; A-AR $P=0.12$; AM-AR $P=0.08, C V P$, A-AM $P=0.51$; A-AR $P=0.97$; AM-AR $P=0.35$.

For within treatments, the differences between procedures (non-abdominal surgery and abdominal surgery) for the A-group were statistically significant $(P<0.05)$ for HR, SYS, DIA, MAP but not for CVP $(P>0.05$, Table 2). For the AM-group differences were statistically significant $(P<0.05)$ for DIA and MAP, but not for HR, SYS, and CVP $(P>0.05$, Table 2). For the AR-group differences between procedures were not statistically significant $(P>0.05)$ for any of the variables (Table 2).

\section{DISCUSSION}

The purpose of this investigation was to evaluate the influence of the anaesthetic technique, i.e. isoflurane anaesthesia on its own or isoflurane anaesthesia supplemented with epidural morphine or ropivacaine on cardiovascular variables. The differences in cardiovascular variables between treatments were not statistically significant; however, the differences between AR- and AM-groups for HR $(P=0.06)$ and $\operatorname{MAP}(P=0.08)$ may be considered as marginally significant. The large variability in data (Fig. 1) could have reduced the ability to detect statistically significant differences between treatments if they did exist. Another factor that could influence outcome of the study is failure of the epidural block. It is not possible to clinically evaluate the effect of the epidural blocks. In this investigation the epidural blocks were administered only by the author, which would minimise inconsistency of the epidural block technique between treatment groups.

When comparing the effects of procedure (non-abdominal and abdominal surgery) during isoflurane anaesthesia, abdominal surgery resulted in a statistically significant change in 4 of the cardiovascular variables (except CVP) during isoflurane only anaesthesia that suggested analgesia could be insufficient despite adequate clinical signs of anaesthesia. When supplementing isoflurane anaes-
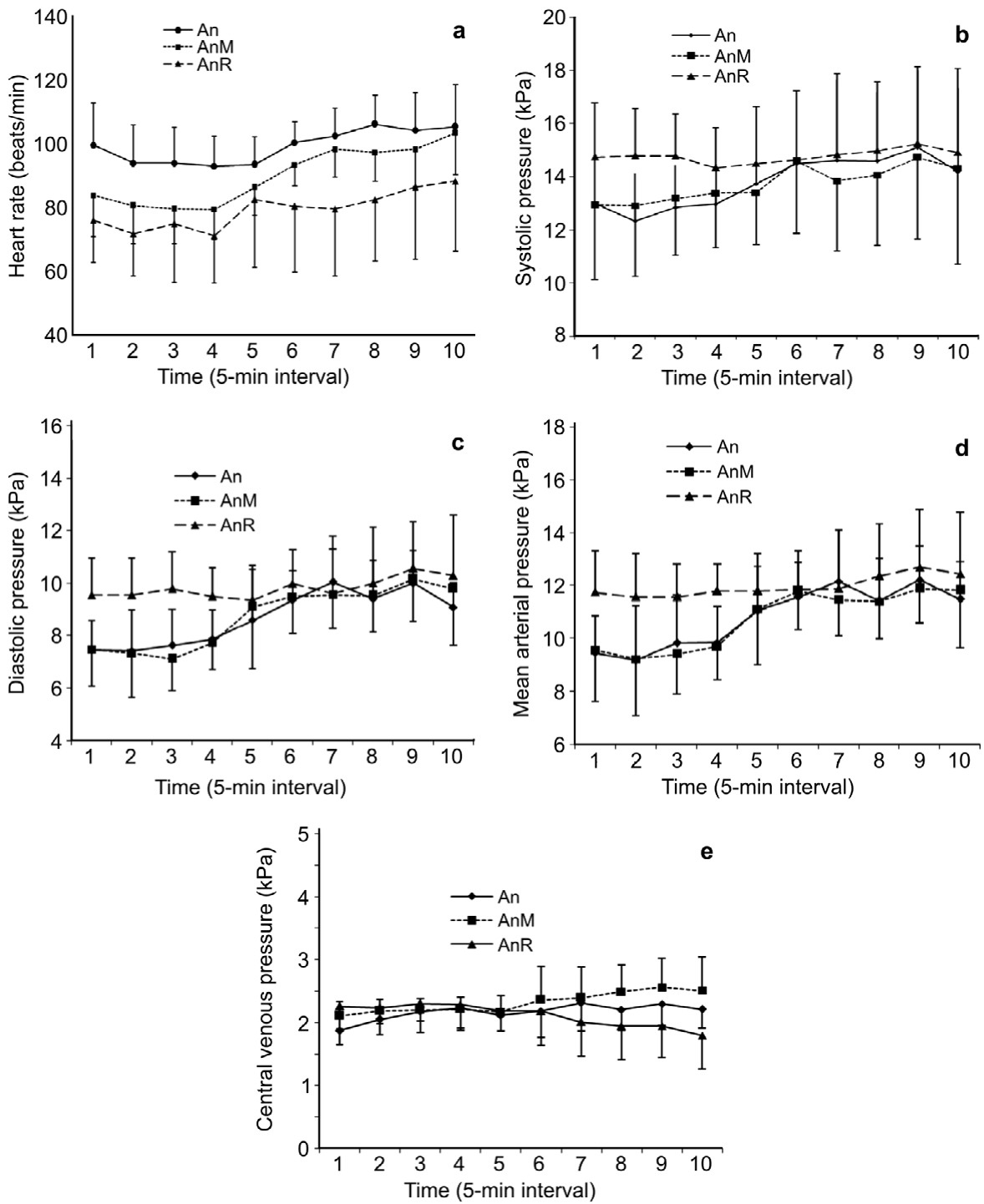

Fig. 1: Mean \pm SD values for (a) heart rate, (b) systolic, $(c)$ diastolic, (d) mean arterial blood pressure, and (e) central venous pressure in isoflurane-anaesthetised pigs during non-abdominal (1-5) and abdominal surgery (6-10). An, anaesthesia; AnM, anaesthesia + epidural morphine; AnR, anaesthesia + epidural ropivacaine.

thesia with epidural morphine, statistically significant changes occurred with 2 of the 4 variables (DIA and MAP) and with ropivacaine epidural no statistically significant changes occurred during abdominal surgery (Table 2). The reduction in the number of statistically significant changes with the morphine epidural and the lack of statistically significant changes in any of the variables with ropivacaine epidural may be of clinical significance in limiting cardiovascular changes during surgery.

In humans, hypotension is an adverse effect associated with epidural anaesthesia $^{1}$ and cardiovascular instability during surgery may be associated with changes in heart rate and arterial blood pres sure $^{22,28}$. The decrease in blood pressure during epidural anaesthesia is the result of a decrease in peripheral vascular resistance ${ }^{1}$. In this investigation the value for MAP was the highest $(12.25 \pm 2.0 \mathrm{kPa})$ during abdominal surgery associated with the ropivacaine epidural anaesthesia (AR-group). This was contrary to the expected effect from ropivacaine as this drug produces peripheral vasodilatation ${ }^{1}$. The delivered isoflurane concentration was decreased from $2.5 \%$ in the A-group to $1.5 \%$ in the AM- and AR-groups, and this may have contributed to the improved blood pressure in the AR-group. The blood pressure in the AM-group was similar to the A-group, which was contrary to the expected effect associated with a lower isoflurane concentration. The cardiovascular effects of isoflurane are dose-dependent and result in a decrease in arterial blood pressure due to a decrease in cardiac contractility and a decrease in peripheral vascular resis tance $^{24}$. The combined effect of isoflurane and epidural anaesthesia may therefore compound peripheral vasodilatation to result in a decrease in arterial blood pressure. The values for MAP during this 
Table 2: Comparison of mean \pm SD cardiovascular variables in isoflurane-anaesthetised pigs supplemented with epidural morphine or epidural ropivacaine during non-abdominal and abdominal surgery.

\begin{tabular}{|c|c|c|c|c|c|c|c|c|c|}
\hline \multirow[t]{2}{*}{ Variable } & \multicolumn{3}{|c|}{ Anaesthesia } & \multicolumn{3}{|c|}{ Anaesthesia + morphine } & \multicolumn{3}{|c|}{ Anaesthesia + ropivacaine } \\
\hline & NonAbd & Abd & $P$ & NonAbd & Abd & $P$ & NonAbd & Abd & $P$ \\
\hline $\mathrm{HR}$ & $82.1 \pm 10.8$ & $98.4 \pm 10.3$ & $0.001^{*}$ & $95.0 \pm 22.2$ & $103.9 \pm 15.4$ & 0.36 & $75.4 \pm 16.4$ & $83.6 \pm 20.6$ & 0.31 \\
\hline SYS & $12.97 \pm 1.53$ & $14.63 \pm 1.77$ & $0.017^{*}$ & $13.17 \pm 2.23$ & $14.3 \pm 2.82$ & 0.36 & $14.62 \pm 1.77$ & $14.91 \pm 2.76$ & 0.78 \\
\hline DIA & $7.78 \pm 1.47$ & $9.58 \pm 1.32$ & $0.002^{*}$ & $7.73 \pm 1.68$ & $9.7 \pm 1.55$ & $0.004^{*}$ & $9.54 \pm 1.28$ & $10.08 \pm 1.82$ & 0.41 \\
\hline MAP & $9.88 \pm 1.83$ & $11.66 \pm 1.34$ & $0.005^{*}$ & $9.81 \pm 1.86$ & $11.68 \pm 1.5$ & $0.01^{*}$ & $11.71 \pm 1.25$ & $12.25 \pm 2.0$ & 0.44 \\
\hline CVP & $2.09 \pm 0.3$ & $2.24 \pm 0.36$ & 0.26 & $2.18 \pm 0.2$ & $2.46 \pm 0.47$ & 0.1 & $2.25 \pm 0.32$ & $1.97 \pm 0.52$ & 0.12 \\
\hline
\end{tabular}

$\mathrm{HR}$, heart rate (beats/min); SYS, systolic pressure $(\mathrm{kPa})$; DIA, diastolic pressure $(\mathrm{kPa})$; MAP, mean arterial pressure $(\mathrm{kPa})$; CVP, central venous pressure $(\mathrm{kPa})$; Abd, abdominal surgery; NonAbd, non-abdominal surgery.

*Statistically significant difference $(P<0.05)$;

investigation $(9.88 \pm 1.83-12.25 \mathrm{kPa})$ were within clinically acceptable limits $(>8.0 \mathrm{kPa})$ and were similar to reported values for pigs $(9.98-13.3 \mathrm{kPa}$, anaesthetic technique not specified $)^{20}$ and during isoflurane anaesthesia $11.1 \pm 3.2 \mathrm{kPa}^{17}$. In ketamine/flunitrazepam-anaesthetised pigs $^{26}$ MAP was higher $(17.3 \mathrm{kPa}) \mathrm{com}$ pared with the highest value observed in this investigation $(12.25 \mathrm{kPa})$ and is probably the result of the ketamine used for maintenance of anaesthesia. Ketamine supports blood pressure by sympathetic activation resulting in an increase in heart rate and blood pressure ${ }^{3}$. Previously published reports indicate a decrease in blood pressure in animals after epidural anaesthesia. In pigs a $30 \%$ decrease in MAP was reported after epidural bupivacaine administration $^{26}$. In isoflurane-anaesthetised dogs the administration of epidural bupivacaine resulted in lower blood pressure values compared with saline placebo or morphine epidural ${ }^{13}$, which is different from the findings in this study where MAP in the AR-group was higher compared to the A- and AM-groups. In this investigation the inspired concentration of isoflurane was reduced in the groups where anaesthesia was supplemented with morphine or ropivacaine and this reduction could improve blood pressure values.

Heart rate was lowest in the AR-group. During abdominal surgery HR increased in all treatment groups but was only statistically significant for the isoflurane anaesthesia group (Table 2). Mean heart rates in this investigation were within range of the reported values for pigs ( $80-130$ beats $/ \mathrm{min})^{20}$ and for isoflurane/ fentanyl-anaesthetised pigs (108 beats/ $\mathrm{min})^{10}$. Isoflurane may also increase heart rate in the $\mathrm{pig}^{24}$. The statistically significant increase in heart rate in the A-group was associated with a statistically significant increase in SYS, DIA and MAP during abdominal surgery and could indicate that analgesia was insufficient during abdominal surgery. During surgery it is not possible to 'standardise' how it is executed, for example differences in blood vessel and liver anatomy between individual animals may result in differences in the intensity of noxious stimulation. The heart rate in the AR-group was lowest and in the AM-group highest during abdominal surgery. The finding of a heart rate that was slightly higher in the AM-group (103.9 beats/min) compared with the A-group (98.4 beats/min) was unexpected as improved analgesia with the morphine epidural was expected. The difference between them is probably clinically insignificant. However, the lower delivered isoflurane concentration $(1.5 \%)$ could possibly be considered to be inappropriate for morphine epidural anaesthesia and should be similar to that of isoflurane $(2.5 \%)$.

Noxious stimulation during surgery results in autonomic activation and changes in cardiovascular variables ${ }^{27}$ and the coadministration of systemic analgesics such as morphine or the epidural administration of local anaesthetics or opioids may be used to reduce autonomic responses to noxious stimulation. In humans, isoflurane on its own is not able to completely suppress autonomic reaction to noxious stimulation ${ }^{6}$. The improved analgesia associated with sympatholysis ${ }^{27}$ was probably responsible for the lower HR observed in the AR-group and could indicate that epidural ropivacaine during isoflurane anaesthesia prevented increases in heart rate and improved blood pressure.

In this investigation a rebreathing anaesthetic system was used that resulted in a decrease in the inhaled anaesthetic concentration $^{4}$. The MAC for isoflurane was reported as $1.65 \% \pm 0.36$ for the tail clamp in the $\mathrm{pig}^{5}$. For surgical anaesthesia with isoflurane an end-tidal concentration of 1.2 X MAC or approximately $2 \%$ will be required. In this investigation the vaporiser was set at $2.5 \%$ (i.e. delivered to the breathing circuit) for the A-group and reduced by $40 \%$ to a delivered isoflurane concentration of $1.5 \%$ where analgesia was supplemented with epidural analgesia. The administration of midazolam and ketamine at induction may contribute to a decrease in the anaesthetic concentration required for surgical anaesthesia ${ }^{14,15}$. Propofol used for tracheal intubation in this investigation may decrease blood pressure but its effect was considered to be minimal as it is an ultra short acting anaesthetic and sufficient time elapsed after its administration and blood pressure recordings ${ }^{11}$. Epidurally administered anaesthetic drugs also reduce the dose for anaesthesia required to produce immobility during surgery ${ }^{2,17}$. As the pedal reflex remained absent and the masseter muscles relaxed in all animals during surgery it may be assumed that the delivered isoflurane concentration was appropriate for the surgical procedures that were performed.

Values for CVP in this investigation (2.09-2.46 kPa) were slightly in excess to reported values for pigs $(1.59-1.86 \mathrm{kPa})^{12}$ and could possibly be ascribed to the generous intravenous administration of balanced electrolyte- and colloid solutions. Changes in CVP during surgery may be the result of blood loss, surgical handling of the liver associated with temporary compression of the portal vein and intravenous fluid administration.

In conclusion, the epidural administration of ropivacaine or morphine in isoflurane-anaesthetised pigs was associated with a decrease in heart rate and improved blood pressure during surgery.

\section{ACKNOWLEDGEMENTS}

This investigation was funded by the National Research Foundation of South Africa. Astra Zenica is thanked for the donation of propofol used in this investigation.

\section{REFERENCES}

1. Borghi B, Casati A, Iuorio S, Celleno D, Michael M, Serafini P, Pusceddu A, Fanelli $\mathrm{G}$, on Behalf of the Study Group on Orthopedic Anesthesia of the Italian Society of Anesthesia, Analgesia, and Intensive Care (SIAARTI) 2002 Frequency of hypotension and bradycardia during general anesthesia, epidural anesthesia, or integrated epiduralgeneral anesthesia for total hip replacement. Journal of Clinical Anesthesia 14: 102-106 
2. Casati L, Fernandez-Galinski S, Barrera E, Pol O, Puig M M 2002 Isoflurane requirements during combined general/epidural anesthesia for major abdominal surgery. Anesthesia \& Analgesia 94: 1331-1337

3. Cook D J, Carton E G, Housmans P R 1991 Mechanism of the positive inotropic effect of ketamine in isolated ferret ventricular papillary muscle. Anesthesiology 74: 880-888

4. Dorsch J A, Dorsch S E 1994 The circle absorption system. In Anon. (eds) Understanding anesthesia equipment: construction, care and complication. Williams \& Wilkens, Baltimore, MD, USA: 191-224

5. Eger 2nd E I, Johnson B H, Weiskopf R B, Holmes M A, Yasuda N, Targ A, Rampil I J 1988 Minimum alveolar concentration of I-653 and isoflurane in pigs: definition of a supramaximal stimulus. Anesthesia \& Analgesia 67: 1174-1176

6. Guignard B 2006 Monitoring analgesia. Best Practice \& Research: Clinical Anaesthesiology 20: $161-180$

7. Gulur P, Nishimori M, Ballantyne J C 2006 Regional anaesthesia versus general anaesthesia, morbidity and mortality. Best Practice \& Research: Clinical Anaesthesiology 20: 249-263

8. Hahnenkamp K, Herroeder S, Hollmann M W 2004 Regional anaesthesia, local anaesthetics and the surgical stress response. Best Practice \& Research: Clinical Anaesthesiology 18: 509-527

9. Holte K, Kehlet H 2002 Epidural anaesthesia and analgesia - effects on surgical stress responses and implications for postoperative nutrition. Clinical Nutrition 10: 536-537

10. Husby P,Heltne JK, Koller ME, Birkeland S, Westby J, Fosse R, Lund T 1998 Midazolamfentanyl-isoflurane anaesthesia is suitable for haemodynamic and fluid balance studies in pigs. Laboratory Animals 323: 3216-3223

11. Ilkiw J E, Pascow P J, Haskins S C, Patz J D 1992 Cardiovascular and respiratory effects of propofol administration in hypovolaemic dogs. American Journal of Veterinary Research 53: 2323-2327

12. Jayaraman S, Khakhar A, Yang H, Bain- bridge D, Quan D 2009 The association between central venous pressure, pneumoperitoneum, and venous carbon dioxide embolism in laparoscopic hepatectomy. Surgical Endoscopy 23: 12-14

13. Kona-Boun J J, Cuvelliez S, Troncy E 2006 Evaluation of epidural administration of morphine or morphine and bupivacaine for postoperative analgesia after premedication with an opioid analgesic and orthopedic surgery in dogs. Journal of the American Veterinary Medical Association 229: 1103-1112

14. Kushiro T, Yamashita K, Umar M A, Maehara S, Wakaiki S, Abe R, Seno T, Tsuzuki K, Izumisawa Y, Muir W W 2005 Anesthetic and cardiovascular effects of balanced anesthesia using constant rate infusion of midazolam-ketamine-medetomidine with inhalation of oxygen-sevoflurane (MKM-OS anesthesia) in horses. Journal of Veterinary Medical Science/Japanese Society of Veterinary Science 67: 379-384

15. Lesser T, Ebner E, Zwiener U 1993 Ketamine-diazepam $\mathrm{N}_{2} \mathrm{O}$ combination anesthesia -a new 'circulatory-system-sparing' type of anesthetic in experimental surgery. Research in Experimental Medicine 193: 207211

16. Lykkegaard K, Lauritzen B, Tessem L, Weikop P, Svendsen O 2005 Local anaesthetics attenuates spinal nociception and HPA-axis activation during experimental laparotomy in pigs. Research in Veterinary Science 79: 245-251

17. Malavasi L M, Jensen-Waern M, Jacobson M, Ryden A, Ohagen P, Nyman G 2006 Effects of extradural morphine on end-tidal isoflurane concentration and physiological variables in pigs undergoing abdominal surgery: a clinical study. Veterinary Anaesthesia \& Analgesia 33: 307-312

18. Moraca R J, Sheldon D G, Thirlby R C 2003 The role of epidural anesthesia and analgesia in surgical practice. Annals of Surgery 238: 663-673

19. Nieuwoudt M, Kunnike R, Smuts M, Becker J, Stegmann G F, Van der Walt C, Neser J, Van der Merwe S 2006 Standardization criteria for an ischemic surgical model of acute hepatic failure in pigs. Biomaterials 27: 3836-3845

20. Riebold T W, Geiser D R, Goodarzi M 1995 Clinical techniques for food animal anesthesia. In Anon. (eds) Large animal anesthesia. Principles and techniques. Iowa State University Press, Ames: 140-173

21. Rodgers A, Walker N, Schug S, McKee A, Kehlet H, van Zundert A, Sage D, Futter M, Saville G, Clark T, MacMahon S 2000 Reduction of postoperative mortality and morbidity with epidural or spinal anaesthesia: results from overview of randomised trials. British Medical Journal 321: 1493-1515

22. Schubert A, Palazzolo J A, Brum J M, Ribeiro M P, Tan M 1997 Heart rate, heart rate variability, and blood pressure during perioperative stressor events in abdominal surgery. Journal of clinical anesthesia 9: 52-60

23. Siniscalchi A, Begliomini B, Matteo G, De Pietri L, Pasetto A 2003 Intraoperative effects of combined versus general anesthesia during major liver surgery. Minerva anestesiologica 69: 885-895

24. Steffey E P, Mama K R 2007 Inhalation anesthetics. In Tranquilli $\mathrm{W}$ J, Thurmon J C, Grimm K A (eds) Lumb \& Jones' veterinary anesthesia and analgesia. Blackwell Publishing, Iowa: 355-393

25. Stegmann G F 2009 Cardiovascular effects of lumbar epidural anaesthesia in isoflurane-anaesthetised pigs during surgical removal of the liver. Journal of the South African Veterinary Association 80: 37-40

26. Vagts D A, Iber T, Szabo B, Haberstroh J Reising K, Puccini M, Geiger K, NoldgeSchomburg G F 2003 Effects of epidural anaesthesia on intestinal oxygenation in pigs. British Journal of Anaesthesia 90: 212 220

27. Veering B T , Cousins M J 2000 Cardiovascular and pulmonary effects of epidural anaesthesia. Anaesthesia $\mathcal{E}$ Intensive Care 28: $620-635$

28. Weissman C 1990 The metabolic response to stress: an overview and update. Anesthesiology 73: 308-327 